\title{
Toll-Like Receptor 9 Is Required for Chronic Stress-Induced Immune Suppression
}

\author{
Hui Li ${ }^{a}$ Jing Zhao a,b Michael Chen ${ }^{a}$ Yang Tan $^{a}$ Xiaohua Yang ${ }^{a}$ Yi Caudle $^{a}$ \\ Deling Yin ${ }^{a}$ \\ a Department of Internal Medicine, College of Medicine, East Tennessee State University, Johnson City, Tenn., USA; \\ b Institute of Developmental Biology, School of Life Sciences, Shandong University, Jinan, China
}

\section{Key Words}

Apoptosis · Chronic stress · Corticosteroids · Immune suppression · TLR9

\begin{abstract}
Objectives: Mental and physical stress can suppress the immune system in both humans and animals. The mechanism by which stress affects immune responses, however, remains poorly defined. Toll-like receptors (TLRs) play a key role in modulating immune responses and cell survival. The mechanisms by which TLRs modulate chronic stress are largely unexplored. Methods: Six- to 8-week-old male mice were subjected to chronic 12-hour daily physical restraint stress. Apoptotic cells were determined by the terminal deoxynucleotidyl transferase-mediated deoxyuridine triphosphate nick end labeling (TUNEL) assay. We examined cytokine levels by enzyme-linked immunosorbent Assay (ELISA). The expression of CYP11A1 was determined by quantitative realtime RT-PCR. Results: TLR9-deficient mice were resistant to chronic stress-induced lymphocyte apoptosis. In addition, in TLR9 knockout (KO) mice, chronic stress-induced upregulation of corticosterone levels was significantly decreased. Notably, lymphocytes from both TLR9 KO and wild-type mice were similarly sensitive to corticosteroid-induced cell apop-
\end{abstract}

tosis. Moreover, TLR9 deficiency blocked the chronic stressinduced imbalance in Thelper (Th) 1 and Th2 cytokine levels. Conclusion: Taken together, our findings reveal that TLR9 plays an essential role in chronic stress-induced immune suppression.

(c) 2013 S. Karger AG, Basel

\section{Introduction}

Different studies with various model systems have revealed that depending on the mood and duration, physical and mental stress could either increase or decrease immune functions in both humans and animals [1-4]. An interaction between stress and immune responses has been studied in various experimental paradigms $[4,5]$. It is well documented that exhausting physical activity and mental stress leads to immunosuppression of the immune system [4-6]. Such suppression of the immune system has strong implications for infectious disease susceptibility and progression $[1,7,8]$, which is at least in part due to lymphocyte apoptosis $[4,9]$. Investigations

H. Li and J. Zhao contributed equally to this work.

\section{KARGER}

E-Mail karger@karger.com

www.karger.com/nim
C) 2013 S. Karger AG, Basel

1021-7401/14/0211-0001\$39.50/0
Assoc. Prof. Deling Yin, PhD

Department of Internal Medicine, College of Medicine

East Tennessee State University

Johnson City, TN 37614 (USA)

E-Mail yin@etsu.edu 
have shown that stress can promote autoimmunity and infectious diseases by influencing the course and outcome of the pathological processes [9-11]. However, acute stress modulates immunoprotection via cell-mediated immunity $[12,13]$. It is well established that acute restraint stress can significantly increase delayed-type hypersensitivity reactions [8], while chronic stress can decrease immune function and enhance susceptibility to diseases $[7,14]$. Stress hormones, including cortisol, play a fundamental role in regulating immune responses and the balance of T helper (Th) 1 and Th2 cytokines, thereby modulating the susceptibility of various immune-related disorders. We observed that chronic restraint stress caused a dramatic decrease in Th 1 cytokine IFN- $\gamma$ and IL-2 levels but an increase in Th2 cytokine IL-4. However, the mechanisms by which Toll-like receptor (TLR) 9 participates in chronic stress-induced immune suppression are unexplored. To define the role of immune responses and stress hormones in mice, a chronic restraint stress murine model has been widely employed [ 5 , 15]. We utilized this model to investigate TLR9-mediated immune responses evaluated by alterations in the number of apoptosis, corticosterone production, and the Th1/Th2 cytokine ratio.

TLRs play an essential role in modulating innate immunity and inflammation as well as cell apoptosis and cell survival [16-18]. There are at least 11 TLRs in mammals. TLR2 detects a broad range of Gram-positive bacterial products, including peptidoglycan, and TLR4 functions as a signal transducer for lipopolysaccharides. TLR4 modulates cell-apoptotic signaling via the interaction of the death domain of MyD88 (myeloid differentiation factor 88) with FADD (Fas-associated death domain) [19]. TLR3, TLR7, and TLR9 are distinct from other TLRs in that they are not expressed on the plasma membrane [18, $20,21]$. TLR9 is identified as a key immune receptor in the TLR family that can recognize bacterial DNA as well as oligodeoxynucleotides containing the CpG motifs responsible for the activating capacity of bacterial DNA (CpG s-oligodeoxynucleotides) [20,21]. Recent studies from us and others have revealed that activation of TLR9 signaling triggers activation of proapoptotic signaling pathways and causes cell apoptosis in various systems [17, $18,20]$. Whether TLR9 participates in chronic stress-induced immune dysfunction is not clear. Therefore, we studied the effect of TLR9 deficiency on chronic stress in TLR9 knockout (KO) and wild-type (WT) mice. Our findings demonstrate a novel role of TLR9 in regulating immune suppression by modulating stress-induced corticosteroid production.

\section{Materials and Methods}

\section{Experimental Animals}

Breeding pairs of TLR9 KO (not a functional KO) mice on a $\mathrm{Balb} / \mathrm{c}$ background were kindly provided by Dr. Shizuo Akira (Osaka University, Osaka, Japan) via Dr. Dennis Klinman (National Cancer Institute, Frederick, Md., USA). Male WT Balb/c mice were purchased from Hallan (Indianapolis, Ind., USA) and maintained in the Division of Laboratory Animal Resources at the East Tennessee State University. The physical restraint procedure was approved by the Institutional Animal Care and Use Committee of the University. All male mice used in the experiments were matched for age and weight.

\section{Physical Restraint Stress}

Six- to 8-week-old male mice were subjected to an established chronic physical restraint protocol used in our laboratory as well as others $[4,5,9,14,22]$. They were placed in a 50 -ml conical centrifuge tube filled with multiple punctures to allow ventilation. Mice were held horizontally in the tubes for $12 \mathrm{~h}$ followed by a 12 hour rest. During the rest period, food and water were provided ad libitum. Control littermates were kept in their original cage, and food and water were provided only during the 12-hour rest. Mice were physically restrained for 1-3 cycles as specified. After physical restraint, mice were sacrificed by $\mathrm{CO}_{2}$ asphyxiation and spleens were harvested.

\section{Detection of Apoptosis by TUNEL Assay}

The frozen spleen sections from unstressed and stressed WT and TLR9 KO mice were harvested for the TUNEL assay (terminal deoxynucleotidyl transferase biotin-dUTP nick end labeling) using an in situ cell death detection kit (Roche Diagnostics, Indianapolis, Ind., USA) according to the manufacturer's instructions and our previous publications $[23,24]$. Splenocytes isolated from WT and TLR9 KO mice were cultured in 96-well plates at $5 \times 10^{5}$ cells $/ \mathrm{ml}$ with or without dexamethasone treatment at different concentrations. After $12 \mathrm{~h}$ of treatment, apoptotic cells were determined by TUNEL assay $[21,23,24]$. The percentage of apoptotic cells was calculated by counting approximately 300 cells.

\section{Measurement of Cytokines by Enzyme-Linked}

Immunosorbent Assay

Splenic lymphocytes from TLR9 KO mice and WT mice were adjusted to a final concentration of $5 \times 10^{5}$ cells $/ \mathrm{ml}$ in 96 -well plates. Lymphocytes were treated with concanavalin A $(5 \mu \mathrm{g} / \mathrm{ml})$. The culture supernatants were harvested after $36 \mathrm{~h}$ of cultivation. The presence of cytokines in the supernatants was determined using cytokine-specific sandwich enzyme-linked immunosorbent assay (ELISA) kits (R\&D Systems, Minneapolis, Minn., USA) according to manufacturer's instructions or as described in our previous publication [5].

\section{Measurement of Corticosterone Levels}

Experimental mice were sacrificed immediately after stress and blood was harvested from each mice. Serum was collected and stored at $-80^{\circ} \mathrm{C}$ until corticosterone determination. Serum corticosterone levels were assessed using a corticosterone ELISA kit (IBL America, Minneapolis, Minn., USA) according to the manufacturer's instructions. 
Isolation of Splenic CD4+ T Cells and Determination of CYP11A1 Expression by Quantitative Real-Time RT-PCR

Splenic CD4+ T cells were negatively selected by using the MagCellect ${ }^{\mathrm{TM}}$ mouse CD4+ T cell isolation kit ( $R \& D$ Systems). Total RNA was isolated from CD4+ T cells by the VERSAGENE ${ }^{\mathrm{TM}}$ RNA tissue kit (Gentra Systems, Minneapolis, Minn., USA), as described previously $[22,25]$. Quantitative real-time RT-PCR was carried out to quantify the expression levels of CYP11A1 on a Bio-Rad iCycler iQ multicolor real-time PCR detection system (Bio-Rad Life Science Research, Hercules, Calif., USA), as reported in our previous studies $[22,25]$. The primers specific for CYP11A1 were described previously [26, 27]. PCR assays were performed in triplicate. The reaction conditions were: $95^{\circ} \mathrm{C}$ for $12 \mathrm{~min}$; followed by 40 cycles at $95^{\circ} \mathrm{C}$ for $15 \mathrm{~s}, 55^{\circ} \mathrm{C}$ for $30 \mathrm{~s}$, and $72^{\circ} \mathrm{C}$ for $30 \mathrm{~s}$. Threshold cycle numbers $\left(C_{\mathrm{T}}\right)$ were determined with a Bio-Rad iCycler iQ multicolor real-time PCR detection system (version 1.1 software) and transformed using the comparative method $\Delta C_{\mathrm{T}}$. Gene-specific expression values were normalized to expression values of GAPDH and/or $\beta$-actin (endogenous control) within each sample. The amount of CYP11A1, normalized to an endogenous reference and relative to a calibrator, was determined by the comparative $C_{\mathrm{T}}$ method $\left(\Delta \Delta C_{\mathrm{T}}\right)$.

\section{Statistical Analysis}

All data were presented as means \pm SEM and analyzed using one-way analysis of variance followed by the Bonferroni test to determine differences among groups. Differences were considered statistically significant for values of $\mathrm{p} \leq 0.05$.

\section{Results}

\section{Chronic Stress-Induced Lymphocyte Reduction \\ Depends on TLR9}

Our previous studies have shown that chronic stress induced splenocyte reduction [5, 14]. However, the mechanisms by which TLRs play a critical role in immune suppression induced by chronic stress are not known yet. To determine the role of TLR9 in chronic stress-induced lymphocyte reduction, we subjected TLR9 KO male mice and their WT control Balb/c male mice to a 12-hour physical restraint daily for 2 days to determined the number of splenocytes $[5,14]$. We found that chronic stress induced lymphocyte reduction in the WT mice, but TLR9 deficiency blocked the stress-induced reduction in lymphocyte numbers (fig. 1). Therefore, TLR9 KO mice lose their sensitivity to the restraint stress-induced reduction in lymphocyte numbers, supporting an important role of TLR9 in stress-induced immune responses.

\section{TLR9 Is Required for Chronic Stress-Induced Lymphocyte Apoptosis}

To investigate whether TLR9 deficiency inhibits the stress-induced lymphocyte reduction via the induction of apoptosis, we assessed apoptotic cells by the TUNEL as-

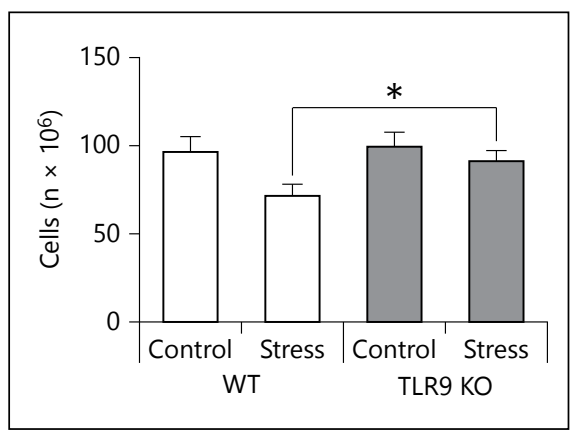

Fig. 1. TLR9 deficiency blocks chronic restraint stress-induced lymphocyte reduction. TLR9 KO and WT Balb/c mice aged 6-8 weeks were subjected to a 12-hour physical restraint daily. After 2 days of stress, mice were sacrificed by $\mathrm{CO}_{2}$ asphyxiation, and the total number of splenocytes was counted (5-7 mice/group; $\left.{ }^{*} \mathrm{p}<0.01\right)$.

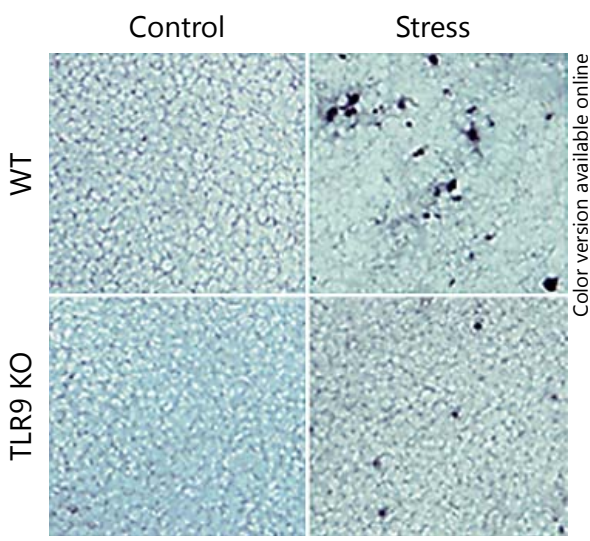

Fig. 2. Chronic stress induces lymphocyte apoptosis through TLR9. WT and TLR9 KO mice aged 6-8 weeks were subjected to a 12-hour physical restraint daily for 2 days. Apoptotic cells in frozen spleen sections were determined by TUNEL assay (data are representative of 3 experiments).

say using histological spleen sections. Our data showed that the number of apoptotic cells was dramatically increased in the spleen of stressed WT mice compared with unstressed control WT mice, whereas no significant difference in apoptotic cell numbers was observed in the spleen of stressed TLR9 KO mice (fig. 2). Therefore, chronic stress induces lymphocyte apoptosis through a TLR9-dependent manner.

\section{TLR9 Deficiency Attenuates Stress-Enhanced Corticosterone Levels}

It has been shown that chronic stress increases the level of corticosterone, a major stress hormone [4, 28]. Enhanced corticosterone levels induce lymphocyte apopto- 


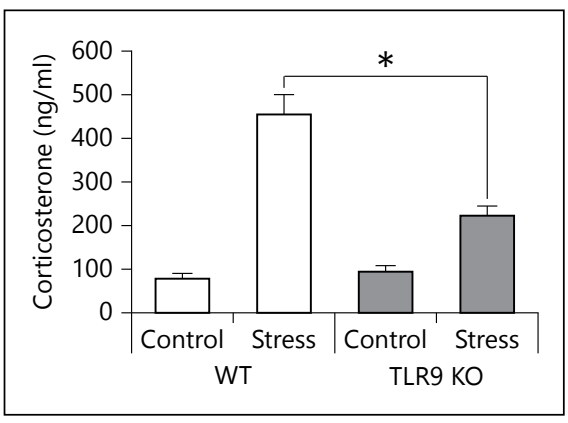

Fig. 3. TLR9 deficiency inhibits chronic stress-induced corticosterone production. WT and TLR9 $\mathrm{KO}$ mice aged 6-8 weeks were subjected to a 12-hour physical restraint daily. After 2 days of stress, blood was harvested and serum was collected for the determination of corticosterone production. Corticosterone levels were examined using a corticosterone ELISA kit (4-6 mice/group; * $\mathrm{p}<0.01)$.

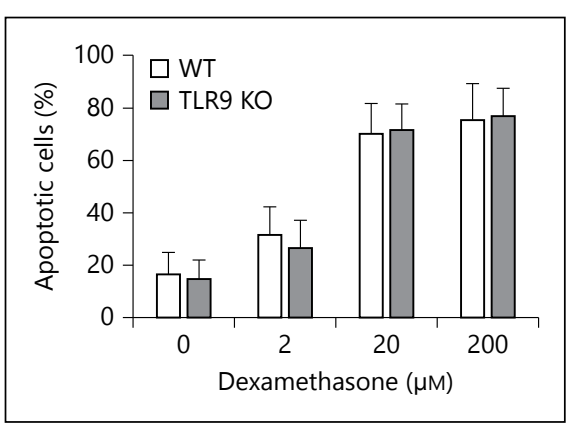

Fig. 4. TLR9 is not necessary for exogenous dexamethasone-induced lymphocyte apoptosis. Single-cell suspensions of splenocytes isolated from TLR9-deficient or WT mice aged 6-8 weeks were incubated in the absence or presence of dexamethasone at different concentrations for $12 \mathrm{~h}$. Apoptotic cells were determined by TUNEL assay. The percentage of apoptotic cells was calculated by counting approximately 300 cells ( 3 independent experiments).

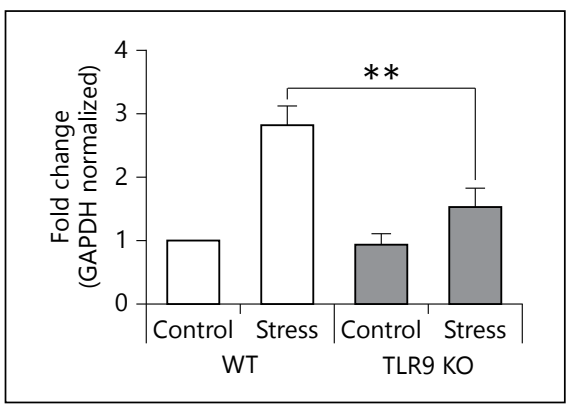

Fig. 5. TLR9 deficiency diminishes chronic stress-induced CYP11A1 expression. WT or TLR9 KO mice aged 6-8 weeks were subjected to $12 \mathrm{~h}$ of physical restraint daily for 2 days. Splenic CD4+ T cells were isolated to determine CYP11A1 expression by real time PCR (4-6 mice/group; $\left.{ }^{* *} \mathrm{p}<0.01\right)$. sis $[4,28]$. We next examined whether TLR9 participates in modulating the glucocorticoid responses following chronic stress; therefore, serum corticosterone levels were determined. Chronic stress significantly enhanced corticosterone levels in the WT mice. Interestingly, a deficiency in TLR9 attenuated stress-enhanced corticosterone levels (fig. 3). Taken together, our results suggest that TLR9 is necessary for the upregulation of the steroid hormone in response to chronic stress.

\section{TLR9 Deficiency Does Not Impair Exogenous}

Dexamethasone-Induced Lymphocyte Apoptosis

TLR9 plays a key role in the upregulation of the steroid hormone induced by stress (fig. 3). We next defined whether TLR9 acts upstream of the corticosterone hormone. Splenocytes from WT and TLR9 KO mice were incubated in the presence or absence of exogenous dexamethasone and then the number of apoptotic cells was determined. Our results revealed no significant differences in apoptotic cell numbers between WT and TLR9 KO mice (fig. 4). These results suggest that steroid-induced cell death is not impaired in TLR9 KO cells, indicating that endogenous TLR9 acts upstream of the corticosterone hormone.

\section{TLR9 Deficiency Diminishes Stress-Increased \\ Expression of CYP11A1}

CYP11A1 (cytochrome P450scc, a cholesterol sidechain cleavage enzyme) plays the first and rate-limiting step in the synthesis of steroid hormones. CYP11A1 is a definitive marker for corticosterone synthesis $[26,27$, 29]. To investigate the effect of TLR9 on CYP11A1 expression during chronic stress, we examined the expression of CYP11A1 in splenic CD4+ T cells in the stressed and unstressed (control) mice. Our studies showed that chronic stress enhanced CYP11A1 expression in the WT mice (fig. 5). Interestingly, chronic stress-enhanced CYP11A1 expression was dramatically attenuated in the TLR9 KO mice. These results suggest that TLR9 plays an important role in corticosterone synthesis during chronic stress.

\section{TLR9 KO Mice Are Resistant to Stress-Induced}

\section{Changes in Th1 and Th2 Cytokine Levels}

Since TLR9 plays an important role in modulating inflammatory responses $[30,31]$, we then examined the effects TLR9 on Th1 and Th2 cytokine production in response to chronic stress. On day 2, Th1 and Th2 cytokines of stressed WT and TLR9 KO mice were assessed from culture supernatants of concanavalin A-stimulated 
Fig. 6. Effect of TLR9 on chronic stress-induced changes in Th1 and Th2 cytokines. TLR9 KO and WT mice aged 6-8 weeks were subjected to a 12-hour physical restraint stress for 2 days. Splenic lymphocytes were treated with concanavalin A $(5 \mu \mathrm{g} / \mathrm{ml})$. After $36 \mathrm{~h}$ of incubation, culture supernatants were harvested. Cytokine production, TNF (a), IL-4 (b), IL-6 (c), MCP-1 (d), and IL-2 (e), in the supernatants was assessed using cytokine-specific sandwich ELISA kits (3-5 mice/group; $* \mathrm{p}<0.01)$.

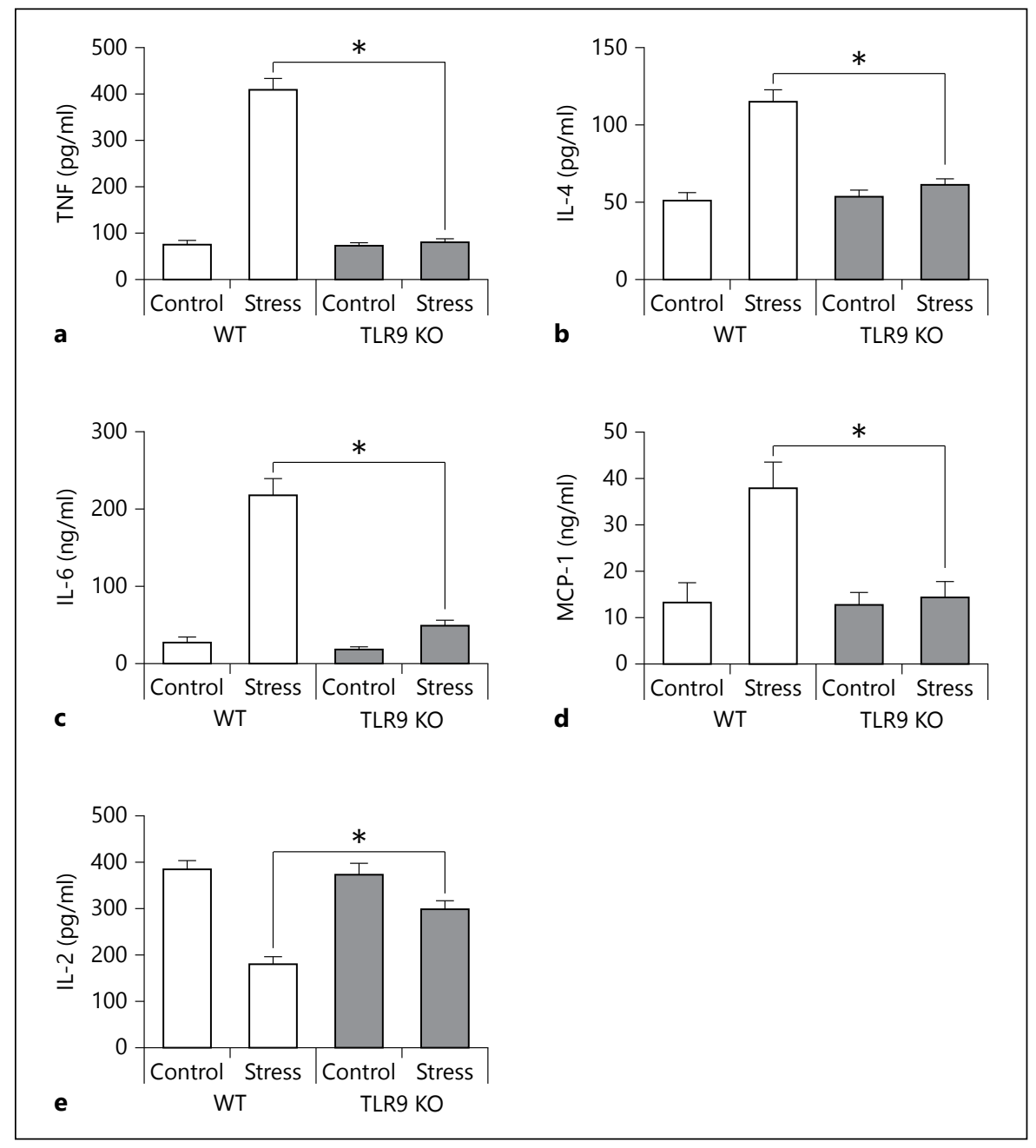

lymphocytes by ELISA. Our data showed that chronic stress significantly enhanced the production of TNF- $\alpha$ (fig. 6a), IL-4 (fig. 6b), IL-6 (fig. 6c), and MCP-1 (fig. 6d) but dramatically inhibited IL-2 production (fig. 6e) in lymphocytes from stressed WT mice compared with lymphocytes from unstressed WT mice. Surprisingly, chronic stress failed to induce these cytokine changes in TLR9 KO mice (fig. 6). Thus, our studies suggest that TLR9 is required for chronic stress-induced immune responses.

\section{Discussion}

It is well established that various stress model systems of physical stress can either enhance or inhibit immune function depending on the type and duration of the stressors $[2,6,7,14]$. Chronic stress-induced alterations in immune responses could result from increased cell death and apoptosis or decreased cell proliferation $[4,5$, 14]. Physical or mental stress promotes the production of stress hormones and neuronal transmitters [15, 32, 33]. However, the role of TLR9 in stress-mediated immune responses has remained unexplored. In the present study, TLR9 was resistant to the chronic restraint stress-induced reduction in the number of lymphocytes. In addition, TLR9 was required for chronic stress-induced lymphocyte apoptosis.

Recent studies have indicated that steroid hormones play an important role in modulating immune functions under chronic stress conditions [4]. It is important to point out that some studies have challenged the general effect of steroids on stress situations [34]. As an example, recent researchers have shown that chronic stress significantly enhances corticosterone production and induces lymphocyte apoptosis, which is at least partially mediated 
by corticosteroids $[4,28]$. We first evaluated whether TLR9 plays a role in modulating glucocorticoid levels under chronic stress conditions; therefore, TLR9 KO and WT mice were subjected to chronic stress. Corticosterone levels were enhanced $>4$-fold in the serum of WT mice following chronic stress. Surprisingly, TLR9 KO mice were resistant to chronic stress-induced upregulation of corticosterone production, suggesting that TLR9 was required for the chronic stress-induced upregulation of steroid hormones. We next defined whether TLR9 affects the expression of CYP11A1, a definitive marker of corticosterone synthesis $[26,27,29]$. Our studies showed that isolated splenic CD4+ T cells from the stressed WT mice increased the expression of CYP11A1 compared with unstressed control mice. Importantly, we found that a deficiency in TLR9 significantly attenuated the chronic stressinduced CYP11A1 expression. We then determined whether TLR9 is involved in exogenous dexamethasoneinduced lymphocyte apoptosis. Our data showed that lymphocytes isolated from TLR9 KO and WT mice did not significantly differ in the percentage of dexamethasone-induced apoptosis, indicating that TLR9 is upstream of corticosterone. These findings suggest that TLR9 plays an important role in the interaction between the neuronal and immune systems. We speculate that TLR9 acts upstream of corticosterone and may be respon- sible for its enhanced levels under chronic stress conditions.

Chronic stress caused an increase in Th2 cytokine production in mice, whereas Th1 cytokine production was decreased. In our current study, we subjected TLR9 KO and WT mice to chronic stress and found that TLR9 deficiency attenuated chronic stress-induced alterations in Th1 and Th 2 cytokine levels. Therefore, the imbalance in Th1 and Th2 cytokines caused by chronic stress apparently required TLR9.

In summary, to the best of our knowledge, our study is the first to reveal that TLR9 is necessary for chronic stressinduced immune suppression by modulating corticosteroid levels. Further studies on TLR9-mediated signaling in response to chronic stress may provide more insights regarding the prevention and/or treatment of stress-induced immune suppression and infectious diseases.

\section{Acknowledgments}

This work was supported in part by research grants NIGMS094740 and NIDA020120 from the National Institutes of Health to D. Yin. The authors wish to express their appreciation to Dr. Shizuo Akira, Osaka University, Osaka, Japan, and Dr. Dennis Klinman, National Cancer Institute, Frederick, Md., USA, for providing breeding pairs of TLR9 KO mice.

\section{References}

1 Shi Y, Devadas S, Greeneltch KM, Yin D, Allan Mufson R, Zhou JN: Stressed to death: implication of lymphocyte apoptosis for psychoneuroimmunology. Brain Behav Immun 2003;17(suppl 1):S18-S26.

2 Stone AA, Bovbjerg DH: Stress and humoral immunity: a review of the human studies. Adv Neuroimmunol 1994;4:49-56.

3 Wilder RL: Neuroendocrine-immune system interactions and autoimmunity. Annu Rev Immunol 1995;13:307-338.

4 Wang KX, Shi Y, Denhardt DT: Osteopontin regulates hindlimb-unloading-induced lymphoid organ atrophy and weight loss by modulating corticosteroid production. Proc Natl Acad Sci USA 2007;104:1477714782.

$\checkmark 5$ Li H, Chen L, Zhang Y, LeSage G, Zhang Y, Wu Y, Hanley G, Sun S, Yin D: Chronic stress promotes lymphocyte reduction through TLR2 mediated PI3K signaling in a $\beta$-arrestin 2 dependent manner. J Neuroimmunol 2011; 233:73-79.

6 Ader R, Cohen N: Psychoneuroimmunology: conditioning and stress. Annu Rev Psychol 1993;44:53-85.
7 Dhabhar FS, McEwen BS: Acute stress enhances while chronic stress suppresses cellmediated immunity in vivo: a potential role for leukocyte trafficking. Brain Behav Immun 1997;11:286-306.

8 Dhabhar FS, McEwen BS: Enhancing versus suppressive effects of stress hormones on skin immune function. Proc Natl Acad Sci USA 1999;96:1059-1064.

-9 Zhang Y, Foster R, Sun X, Yin Q, Li Y, Hanley G, Stuart C, Gan Y, Li C, Zhang Z, Yin D: Restraint stress induces lymphocyte reduction through p53 and PI3K/NF- $\mathrm{KB}$ pathways. J Neuroimmunol 2008;200:71-76.

10 Sheridan JF, Dobbs C, Jung J, Chu X, Konstantinos A, Padgett D, Glaser R: Stress-induced neuroendocrine modulation of viral pathogenesis and immunity. Ann NY Acad Sci 1998;840:803-808.

11 Shanks N, Kusnecov AW: Differential immune reactivity to stress in BALB/CByJ and C57BL/6J mice: in vivo dependence on macrophages. Physiol Behav 1998;65:95-103.

12 Dhabhar FS, McEwen BS: Stress-induced enhancement of antigen-specific cell-mediated immunity. J Immunol 1996;156:2608-2615.
13 Nash MS: Exercise and immunology. Med Sci Sports Exerc 1994;26:125-127.

14 Li H, Smalligan DA, Xie N, Javer A, Zhang Y, Hanley G, Yin D: $\beta$-Arrestin 2-mediated immune suppression induced by chronic stress. Neuroimmunomodulation 2011;18: 142-149.

15 Wang KX, Shi YF, Ron Y, Kazanecki CC, Denhardt DT: Plasma osteopontin modulates chronic restraint stress-induced thymus atrophy by regulating stress hormones: inhibition by an anti-osteopontin monoclonal antibody. J Immunol 2009;182:2485-2491.

16 Aderem A, Ulevitch RJ: Toll-like receptors in the induction of the innate immune response. Nature 2000;406:782-787.

17 He L, Li H, Chen L, Miao J, Jiang Y, Zhang Y, Xiao Z, Hanley G, Li Y, Zhang X, LeSage G, Peng Y, Yin D: Toll-like receptor 9 is required for opioid-induced microglia apoptosis. PLoS One 2011;6:e18190.

18 Chen L, Shi W, Li H, Sun X, Fan X, LeSage G Li H, Li Y, Zhang Y, Zhang X, Zhang Y, Yin D: Critical role of toll-like receptor 9 in morphine and Mycobacterium tuberculosis-induced apoptosis in mice. PLoS One 2010;5:e9205. 
19 Haase R, Kirschning CJ, Sing A, Schrottner P, Fukase K, Kusumoto S, Wagner H, Heesemann J, Ruckdeschel K: A dominant role of Toll-like receptor 4 in the signaling of apoptosis in bacteria-faced macrophages. J Immunol 2003;171:4294-4303.

-20 Liang X, Moseman EA, Farrar MA, Bachanova $\mathrm{V}$, Weisdorf DJ, Blazar BR, Chen W: Tolllike receptor 9 signaling by CpG-B oligodeoxynucleotides induces an apoptotic pathway in human chronic lymphocytic leukemia B cells. Blood 2010;115:5041-5052.

21 Plitas G, Burt BM, Nguyen HM, Bamboat ZM, DeMatteo RP: Toll-like receptor 9 inhibition reduces mortality in polymicrobial sepsis. J Exp Med 2008;205:1277-1283.

22 Yin D, Zhang Y, Stuart C, Miao J, Zhang Y, Li C, Zeng X, Hanley G, Moorman J, Yao Z, Woodruff M: Chronic restraint stress modulates expression of genes in murine spleen. J Neuroimmunol 2006;177:11-17.

-23 Yang X, Zhou G, Ren T, Li H, Zhang Y, Yin D, Qian H, Li Q: $\beta$-Arrestin prevents cell apoptosis through pro-apoptotic ERK1/2 and p38 MAPKs and anti-apoptotic Akt pathways. Apoptosis 2012;17:1019-1026.
24 Li H, Sun X, LeSage G, Zhang Y, Liang Z, Chen J, Hanley G, He L, Sun S, Yin D: $\beta$-Arrestin 2 regulates Toll-like receptor 4 -mediated apoptotic signalling through glycogen synthase kinase-3 $\beta$. Immunology 2010;130:556-563.

25 Li Y, Li H, Zhang Y, Sun X, Hanley GA, LeSage G, Zhang Y, Sun S, Peng Y, Yin D: Toll-like receptor 2 is required for opioidsinduced neuronal apoptosis. Biochem Biophys Res Commun 2010;391:426-430.

26 Oka H, Emori Y, Hayashi Y, Nomoto K: Breakdown of Th cell immune responses and steroidogenic CYP11A1 expression in CD4+ $T$ cells in a murine model implanted with B16 melanoma. Cell Immunol 2000;206:7-15.

27 Qiao S, Chen L, Okret S, Jondal M: Age-related synthesis of glucocorticoids in thymocytes. Exp Cell Res 2008;314:3027-3035.

28 Calcagni E, Elenkov I: Stress system activity, innate and $\mathrm{T}$ helper cytokines, and susceptibility to immune-related diseases. Ann NY Acad Sci 2006;1069:62-76.
29 Vacchio MS, Papadopoulos V, Ashwell JD: Steroid production in the thymus: implications for thymocyte selection. J Exp Med 1994;179:1835-1846.

30 Baeuerle PA, Baltimore D: NF- $\kappa$ B: ten years after. Cell 1996;87:13-20.

31 Wang Y, Tang Y, Teng L, Wu Y, Zhao X, Pei G: Association of $\beta$-arrestin and TRAF6 negatively regulates Toll-like receptor-interleukin 1 receptor signaling. Nat Immunol 2006;7: 139-147.

32 Dhabhar FS: Stress-induced augmentation of immune function - the role of stress hormones, leukocyte trafficking, and cytokines. Brain Behav Immun 2002;16:785-798.

33 Dhabhar FS, Satoskar AR, Bluethmann H, David JR, McEwen BS: Stress-induced enhancement of skin immune function: a role for $\gamma$ interferon. Proc Natl Acad Sci USA 2000;97:2846-2851.

34 Minton JE: Function of the hypothalamic-pituitary-adrenal axis and the sympathetic nervous system in models of acute stress in domestic farm animals. J Anim Sci 1994;72: $1891-1898$ 